\title{
Serum creatinine levels in relationship with mean platelet volume in patients with chronic kidney disease

\author{
Mohammad Reza Tamadon ${ }^{1}$, Sayed Mohammad-Ali Torabi ${ }^{1}$, Jamileh Moghimi ${ }^{1}$, Majid \\ Mirmohammadkhani $^{2}$, Farahnaz Ghahremanfard ${ }^{3 *}$
}

${ }^{1}$ Department of Internal Medicine, Semnan University of Medical Sciences, Semnan, Iran

${ }^{2}$ Social Determinants of Health Research Center, Semnan University of Medical Sciences, Semnan, Iran

${ }^{3}$ Cancer Research Center, Semnan University of Medical Sciences, Semnan, Iran

\section{A R T I C L E I N F O}

\section{Article Type:}

Brief Communication

\section{Article History:}

Received: 20 May 2017

Accepted: 14 August 2017

Published online: 30 August 2017

\section{Keywords:}

Chronic kidney disease

Mean platelet volume

Creatinine

Hypertension

Glomerular filtration rate

\begin{abstract}
A B S T R A C T
Introduction: Chronic kidney disease (CKD) is a progressive decline in glomerular filtration rate (GFR) which is mainly caused by diabetic nephropathy and hypertension.

Objectives: This study evaluated the relationship between changes in mean platelet volume (MPV) and serum creatinine levels.

Patients and Methods: In this retrospective study, medical records of 100 patients with CKD including demographics data, medications, past-history, serum creatinine levels, and MPV were gathered. Additionally, we excluded patients who had a history of taking aspirin, idiopathic thrombocytopenic purpura, preeclampsia, sepsis, disseminated intravascular coagulation, aplastic anemia and administration of cytotoxic drugs.

Results: This study was conducted on 100 CKD patients. Relationship between changes in MPV with serum creatinine level was evaluated by adjusting for gender, age, CKD stages, etiology, and weight. The changes in MPV and serum creatinine level were not significant with adjusting by underlying variables. However, in patients with high blood pressure, relationship of serum creatinine levels with MPV was inversely significant $(r=-0.484, P=0.049)$.

Conclusion: In CKD patients with high blood pressure, the changes in serum creatinine level have an inverse relationship with MPV. However, MPV is not an appropriate indicator for predicting of creatinine changes.
\end{abstract}

Implication for health policy/practice/research/medical education:

In a study on $100 \mathrm{CKD}$ patients, with hypertension, we found that changes in serum creatinine level have an inverse relationship with MPV.

Please cite this paper as: Tamadon MR, Torabi SMA, Moghimi J, Mirmohammadkhani M, Ghahremanfard F. Serum creatinine levels in relationship with mean platelet volume in patients with chronic kidney disease. J Renal Inj Prev. 2018;7(1):3841. DOI: 10.15171/jrip.2018.09.

\section{Introduction}

Chronic kidney disease $(\mathrm{CKD})$ is a progressive and irreversible deterioration of the renal excretory function and glomerular filtration rate (GFR) that results in implementation of renal replacement therapy (dialysis or renal transplant). The most common causes of CKD are diabetic nephropathy caused by type 2 diabetes mellitus and high blood pressure (1). Around 27 million people or $15 \%$ of US adults are estimated to have CKD. According to the high prevalence of CKD, it is important to evaluate and screen patients at risk and to identify the predisposing factors and complications of CKD (1).
Diabetes mellitus and high blood pressure are the most important risk factors. On the other hand, cardiovascular disease and stroke are the most important complications of CKD $(2,3)$. Evaluation of GFR is the best indicator of renal function. GFR changes by age, sex, and body mass index. GFR less than $60 \mathrm{~mL} / \mathrm{min} / 1.73 \mathrm{~m}^{2}$ means the person has early kidney disease (4). GFR is often estimated by measurement of creatinine clearance in a 24hour urine collection (4). Creatinine is a product of the metabolism of muscle cells and is constantly produced and its level depends on muscle mass. Serum creatinine level is an important indicator of kidney function. It has 
low rates of reabsorption and secretion, therefore its level can be easily measured. Serum creatinine level increases in the conditions of significant damage to the nephrons. Therefore it is not a proper test for diagnosis of early stages of kidney failure (5).

Numerous studies have evaluated the relationship between increases in serum creatinine and other indicators to find early diagnosis and treatment for CKD $(6,7)$. Some studies have recently been focused on the relationship between serum creatinine and mean platelet volume (MPV) (810). MPV is an indicator which is used for measuring the activity of the bone marrow and structural problems of platelets. MPV depends on the production quality of platelets in bone marrow and is not related to aging phenomenon in blood circulation. MPV is affected by some conditions such as idiopathic thrombocytopenic purpura, preeclampsia, sepsis, disseminated intravascular coagulation, aplastic anemia, taking cytotoxic agents, and some inherited disorders including BernardSoulier syndrome, and Wiskott-Aldrich syndrome (11). Turgutalp et al showed that MPV in diabetic patients was higher than that in normal participants and MPV had a direct relationship with creatinine and proteinuria. They also found an inverse relationship of MPV with GFR (10). In a study by Ju et al, MPV had an inverse correlation with GFR in patients with CKD. In addition, it was significantly increased with the progression of CKD $(8,12)$. CKD leads to irreversible reduction in kidney function and in the end-stages of the disease, patients need to renal replacement therapy. Therefore, its diagnosis in the early stages, can remarkably reduce health care costs (5). MPV is one of the available tests which is affected by changes in serum creatinine. It is assumed that it can detect the progression of CKD in its earlier stages.

\section{Objectives}

This study aims to evaluate the possible relationship between MPV and serum creatinine and find its clinical implication.

\section{Patients and Methods \\ Study population}

In a retrospective cross-sectional study, all patients with CKD who referred to the nephrology clinic in Kosar hospital in Semnan enrolled to the study. Study was conducted between 2013 and 2016. We excluded patients who had a history of taking aspirin, idiopathic thrombocytopenic purpura, preeclampsia, sepsis, disseminated intravascular coagulation, aplastic anemia, taking cytotoxic drugs, Bernard-Soulier syndrome, and Wiskott-Aldrich syndrome and patients under dialysis.

\section{Laboratory assessments}

Medical records of eligible patients were reviewed and the required data including demographics data, medications, past-history, serum creatinine levels, and MPV was gathered.

\section{Ethical considerations}

This research was performed following the Declaration of Helsinki principles. The Ethics Committee of Semnan University of Medical Sciences approved the study. All information about individuals was coded and kept confidential.

\section{Statistical analysis}

Descriptive statistical methods were used to compare the means. Since changes in MPV and also creatinine did not have normal distribution (Kolmogorov-Smirnov test, $P=0.001$ ), we calculated the median and interquartile range by gender, age, weight and stages of CKD. Nonparametric Spearman's correlation coefficient was used to analyze the correlation between the variables. Multiple regression analysis was applied too. The significance level was set at $P<0.05$. We used SPSS software (version 20) for data analyzing.

\section{Results}

One hundred CKD patients not yet on dialysis were enrolled in the study. Of which 28 were female. Their mean age was $71.25 \pm 13.85$ years. CKD patients were classified based on serum creatinine level. Around 45 patients were in stage three, 48 patients in stage 4 , and 7 patients were in stage five. The most common underlying disease was diabetes mellitus ( 31 patients) which was followed by concomitant presence of diabetes and high blood pressure (18 patients), high blood pressure (17 patients), and nephrotic syndrome (2 patients). In addition, 32 patients had unknown underlying condition. Tables 1 and 2 represent the data obtained from the medical records. In this study, patients with underlying high blood pressure had a significant negative relationship of MPV and serum creatinine ( $r=-0.484, P=0.049)$. We did not observe any relationship between serum creatinine levels and MPV after adjusting the variables including age, gender, weight and underlying diseases $(P>0.05)$.

\section{Discussion}

This study showed that in CKD patients with hypertension, serum creatinine inversely correlated with MPV. Turgutalp et al evaluated MPV and related risk factors in patients with various stages of diabetic nephropathy. They estimated MPV in healthy participants (group 1, n=157), patients with type 2 diabetes mellitus without complaint (group 2, $\mathrm{n}=160$ ), diabetic patients with proteinuria (group 3, n=140), and patients with CKD caused by diabetic nephropathy (group 4, $\mathrm{n}=160$ ). Based on their results, MPV in diabetic patients was higher than that of normal participants. They concluded that MPV had a direct relationship with proteinuria and had an inverse relationship with GFR (10). The results of the mentioned study were similar to our results. In our study an inverse relationship between serum creatinine and MPV in CKD patients with hypertension was detected.

Ju et al conducted a retrospective study to evaluate the 
Table 1. Data of patients

\begin{tabular}{|c|c|c|c|c|c|}
\hline Variable & Mean & Median & SD & Minimum & Maximum \\
\hline Age (y) & 71.35 & 74.00 & 13.85 & 33 & 93 \\
\hline Weight (kg) & 64.48 & 64.50 & 12.64 & 35 & 116 \\
\hline Creatinine $1(\mathrm{mg} / \mathrm{dL})^{*}$ & 1.920 & 1.750 & 0.86 & 1.00 & $6.30 / 6$ \\
\hline Creatinine $2(\mathrm{mg} / \mathrm{dL})^{*}$ & 1.99 & 1.70 & 1.02 & 1.00 & 6.20 \\
\hline MPV $1(\mathrm{fL})^{*}$ & 8.68 & 8.65 & 1.61 & 3.90 & 12.60 \\
\hline MPV $2(\mathrm{fL})^{*}$ & 9.29 & 8.70 & 6.37 & 4.20 & 70.60 \\
\hline $\mathrm{GFR}\left(\mathrm{ml} / \mathrm{min} / \mathrm{m}^{2}\right)$ & 29.30 & 29.00 & 9.13 & 8.00 & 45.00 \\
\hline Changes in MPV & 0.61 & 0.10 & 6.12 & -4.00 & 60.10 \\
\hline Changes in creatinine & 0.68 & 0.00 & 0.65 & -2.20 & 2.50 \\
\hline
\end{tabular}

* MPV1: Baseline measurement, MPV2: measurement after progression of disease, creatinine 1; Baseline measurement, creatinine 2; measurement after progression of CKD.

Table 2. Median and Interquartile range of changes in MPV and serum creatinine based on gender, age, and weight and also CKD stage

\begin{tabular}{|c|c|c|c|c|c|}
\hline \multirow{2}{*}{ Variable } & & \multicolumn{2}{|c|}{ Changes in MPV } & \multicolumn{2}{|c|}{ Changes in creatinine } \\
\hline & & Median & Interquartile range & Median & Interquartile range \\
\hline \multirow{2}{*}{ Gender } & Male & -0.1 & 1.42 & 0.05 & 0.75 \\
\hline & Female & 0.2 & 0.97 & 0.00 & 0.58 \\
\hline \multirow{2}{*}{ Age group (year) } & $>70$ & 0.1 & 0.15 & -0.1 & 0.78 \\
\hline & $\leq 70$ & 0.1 & 0.85 & 0.1 & 0.58 \\
\hline \multirow{2}{*}{ Weight (kg) } & $>65$ & 0.00 & 1.05 & 0.00 & 0.70 \\
\hline & $\leq 65$ & 0.1 & 1.03 & 0.00 & 0.60 \\
\hline \multirow{3}{*}{ CKD stage } & 3 & 0.00 & 1.07 & 0.00 & 0.3 \\
\hline & 4 & 0.2 & 0.77 & 0.00 & 0.98 \\
\hline & 5 & -0.1 & 1.80 & -0.2 & 1.90 \\
\hline
\end{tabular}

relationship between MPV and GFR in patients with CKD. In this study, MPV had a negative relationship with estimated GFR in patients with CKD. Their study showed, parallel with progression of CKD, MPV was significantly increased (8). Our study showed an inverse relationship between MPV and creatinine level in patients with hypertension and we did not observe any relationship between creatinine levels (as an indicator for progression of kidney disease) and MPV after adjusting the variables including age, gender, and weight and also underlying diseases except hypertension.

In our study, we did not observe any significant correlation between the stage of CKD and changes in MPV. However, in patients with high blood pressure, the changes in MPV were inversely related to the progression of kidney disease. Perhaps, this marker can be applicable to predict the progression of kidney disease toward the chronic states of the disease in patients with high blood pressure, while more studies are necessary to prove it.

\section{Conclusion}

This study showed that the changes in MPV had an inverse relationship with changes in creatinine levels only in patients with underlying hypertension $(r=-0.484$, $P=0.049)$. Thus, MPV cannot be a good prognostic factor to predict the progression of kidney disease. However, more studies are needed to be conducted in this field.

Limitations of the study

Study was retrospective study. It is recommended to conduct prospective studies to investigate the relationship between creatinine and MPV. Moreover, it is also suggested to carry out further studies with larger sample sizes on patients comparable in terms of sex and body mass index.

\section{Authors' contribution}

MRT and FG; contributed to study design, preparation of manuscript and final revision. JM; acted as the consultant of study. SMAT; performed data gathering. MM; helped in data analysis and interpretation. All authors read and approved the paper.

\section{Conflicts of interest}

The authors declare no conflict of interest.

Ethical considerations

Ethical issues (including plagiarism, data fabrication, double publication) have been completely observed by the authors.

\section{Funding/Support}

This article is extracted from general practitioner thesis of Sayed Mohammad Ali Torabi, was supported by ViceChancellor for Research Affairs, Semnan University of Medical Sciences.

\section{References}

1. Kopple JD. National kidney foundation K/DOQI clinical practice guidelines for nutrition in chronic kidney disease. Am J Kidney Dis. 2001; 37:S66-70. 
2. Becherucci F, Roperto RM, Materassi M, Romagnani P. Chronic kidney disease in children. Clin Kidney J. 2016; 9: 583-91. doi: 10.1093/ckj/sfw047.

3. Snyder S, Pendergraph B. Detection and evaluation of chronic kidney disease. Am Fam Physician. 2005;72:172332 .

4. Stevens LA, Coresh J, Greene T, Levey AS. Assessing kidney function-measured and estimated glomerular filtration rate. N Engl J Med. 2006; 354:2473-83. doi: 10.1056/ NEJMra054415.

5. Taylor EH. Clinical Chemistry. New York: John Wiley and Sons; 1989:58-62.

6. Asnani MR, Reid ME. Determining glomerular filtration rate in homozygous sickle cell disease: utility of serum creatinine based estimating equations. PLoS One. 2013;8:e69922. doi: 10.1371/journal.pone.0069922.

7. Siddappa JK, Singla S, Mohammed Al Ameen S, Kumar N. Correlation of ultra-sonographic parameters with serum creatinine in chronic kidney disease. J Clin Imaging Sci. 2013;3:28. doi: 10.4103/2156-7514.114809.

8. Ju HY, Kim JK, Hur SM, Woo SA, Park KA, Park MY, et al. Could mean platelet volume be a promising biomarker of progression of chronic kidney disease? Platelets. 2015;26:143-7. doi: 10.3109/09537104.2014.890179.

9. Yenigun EC, Aypak C, Turgut D, Piskinpasa SV, Cevher SK, $\mathrm{Koc} \mathrm{E}$, et al. Is there a relation between mean platelet volume and chronic kidney disease stages in diabetic patients? Int J Clin Exp Med. 2016;9:330-5.

10. Turgutalp K, Özhan O, Akbay E, Tombak A, Tiftik N, Ozcan $\mathrm{T}$, et al. Mean platelet volume and related factors in patients at different stages of diabetic nephropathy: a preliminary study. Clin Appl Thromb Hemost. 2014;20:190-5. doi: 10.1177/1076029612456734.

11. Liu S, Ren J, Han G, Wang G, Gu G, Xia Q, et al. Mean platelet volume: a controversial marker of disease activity in Crohn's disease. Eur J Med Res. 2012;17:27. doi: 10.1186/2047-783X-17-27.

12. Sharpe PC, Desai ZR, Morris TC. Increase in mean platelet volume in patients with chronic renal failure treated with erythropoietin. J Clin Pathol 1994;47:159-161. doi: 10.1136/ jcp.47.2.159

Copyright (c) 2018 The Author(s); Published by Nickan Research Institute. This is an open-access article distributed under the terms of the Creative Commons Attribution License (http://creativecommons.org/licenses/by/4.0), which permits unrestricted use, distribution, and reproduction in any medium, provided the original work is properly cited. 